\title{
Spontaneous Symmetry Breaking for Scalar QED with Non-minimal Chern-Simons Coupling
}

\author{
D.S. Irvine ${ }^{a}$, M.E. Carrington ${ }^{b, c}$, G. Kunstatter ${ }^{c, d}$ and D. Pickering ${ }^{a}$ \\ ${ }^{a}$ Department of Mathematics and Computer Science, Brandon University, Brandon, Manitoba, \\ R7A 6A9 Canada \\ ${ }^{b}$ Department of Physics, Brandon University, Brandon, Manitoba, R7A 6A9 Canada \\ ${ }^{c}$ Winnipeg Institute for Theoretical Physics, Winnipeg, Manitoba, R3B 2E9 \\ ${ }^{d}$ Department of Physics, University of Winnipeg, Winnipeg, Manitoba, R3B 2E9 Canada
}

\begin{abstract}
We investigate the two-loop effective potential for both minimally and nonminimally coupled Maxwell-Chern-Simons theories. The non-minimal gauge interaction represents the magnetic moment interaction between a charged scalar and the electromagnetic field. In a previous paper we have shown that the two loop effective potential for this model is renormalizable with an appropriate choice of the non-minimal coupling constant. We carry out a detailed analysis of the spontaneous symmetry breaking induced by radiative corrections. As long as the renormalization point for all couplings is chosen to be the true minimum of the effective potential, both models predict the presence of spontaneous symmetry breaking. Two loop corrections are small compared to the one loop result, and thus the symmetry breaking is perturbatively stable.
\end{abstract}

\section{INTRODUCTION}

Maxwell-Chern-Simons electrodynamics has been studied extensively in recent years for a variety of reasons. The Chern-Simons term gives the photon a topological mass without spontaneously breaking gauge symmetry [1] and allows for the existence of charged particles with fractional statistics [2]. Pure Chern-Simons scalar electrodynamics admits topological and non-topological self-dual solitons, for which many exact solutions to the classical equations of motion are available [3]. Such theories may have physical significance. Relativistic three dimensional Chern-Simons theories provide a consistent description of the high temperature limit of four dimensional gauge theories [4], and certain solid state systems with planar dynamics [2]. In addition, the non-relativistic version of Maxwell-Chern-Simons theory has been applied to the fractional Hall effect, and more recently to rotating superfluid ${ }^{3} \mathrm{He}-\mathrm{A}[$ [5].

Work has also been done with a version of scalar electrodynamics in three dimensions in which a non-minimal Chern-Simons type gauge interaction is introduced [6, 7]. The nonminimal coupling in this model represents a magnetic moment interaction between the charged scalar and the electromagnetic field. It is of interest for several reasons. Firstly, 
it is well known that one of the most important features of scalar quantum electrodynamics (QED) is the occurrence of the Coleman-Weinberg mechanism [8]. In scalar QED with non-minimal coupling, the Chern-Simons term is generated through the Coleman-Weinberg mechanism [6]. In this sense, the non-minimal model is the one in which the Chern-Simons term arises naturally rather than being put in by hand.

Another reason that the non-minimal model is of interest involves the study of vortex solutions. In recent years, the classical vortex solutions of 2+1-dimensional Chern-Simons field theories have received considerable attention [3, 9]. To find such a solution exactly, the model must be self-dual. A self-dual theory is one in which the classical equations of motion can be reduced from second- to first-order differential equations. In the absence of a Maxwell term, scalar QED with a Chern-Simons term is self-dual, and the topological and non-topological vortex solutions have been found with an appropriately chosen scalar potential [3]. However, if the Maxwell term is present, a self-dual Maxwell-Chern-Simons theory can be achieved only if a magnetic moment interaction between the scalar and the gauge field, i.e. the non-minimal Chern-Simons coupling, is introduced [9, 10].

It is well known that Maxwell-Chern-Simons scalar QED is renormalizable. Non-minimal gauge interactions are, however, notoriously non-renormalizable in four dimensions. There is some hope that the situation might be different in three dimensions. Some time ago it was found by two of us that the non-minimal Chern-Simons coupling in 2+1-dimensional scalar electrodynamics is actually renormalizable at the one-loop level [6]. The renormalizability occurs because the non-minimal gauge interaction contains the three-dimensional antisymmetric tensor. At the two loop level, the full effective action of the non-minimal theory is not renormalizable. However, it has been shown by two of us that the two loop effective potential is renormalizable providing certain conditions are satisfied by the coupling constants of the model [11]. When the lowest order in the momentum expansion is sufficient, the effective potential can be used to obtain physical information about spontaneous symmetry breaking at the two-loop level.

In this paper, we compare the symmetry breaking phase transitions in the minimal and non-minimal models at two loop order. The two-loop behaviour of the minimal model has recently been analyzed in detail [12]. We discuss the calculation of the effective potential for this model in section II. Section III contains a brief review of the calculation of the effective potential for the non-minimal model. Details are given in [11]. In section IV we discuss the renormalization of the minimal effective potential. In contrast to [12] we show that one obtains perturbatively reliable spontaneous symmetry breaking as long as the minimum of the potential is used to provide a physical renormalization point. In section $\mathrm{V}$ we discuss the renormalization of the non-minimal model. In sections IV and V calculations are carried out using Mathematica. Conclusions are presented in section VI.

\section{SCALAR QED WITH MINIMAL CHERN-SIMONS COUPLING}

\section{A. The Effective Potential}

The minimal model has been studied in a previous paper [12]. The Lagrangian for scalar QED in $2+1$ dimensions with a minimal Chern-Simons coupling is, 


$$
\begin{gathered}
\mathcal{L}=\frac{1}{4} F_{\mu \nu} F^{\mu \nu}-\frac{\kappa}{2} \epsilon^{\mu \nu \rho} A_{\mu} \partial_{\nu} A_{\rho}+\mathcal{L}_{g . f .}+\mathcal{L}_{F . P .}+\frac{1}{2}\left|D_{\mu} \phi\right|^{2} \\
-\frac{m^{2}}{2}\left(\phi^{*} \phi\right)-\frac{\lambda}{4 !}\left(\phi^{*} \phi\right)^{2}-\frac{\nu}{6 !}\left(\phi^{*} \phi\right)^{3}
\end{gathered}
$$

where $D_{\mu}=\partial_{\mu}+i e A_{\mu}$ and $\phi=\chi+i \eta$. In the $R_{\xi}$ gauge we have,

$$
\begin{aligned}
& \mathcal{L}_{\text {g.f. }}=-\frac{1}{2 \alpha}\left(\partial_{\mu} A^{\mu}-\alpha e v \eta\right)^{2} \\
& \mathcal{L}_{F . P .}=-c^{\dagger}\left(\partial^{2}+\alpha e^{2} v \chi\right) c .
\end{aligned}
$$

We consider first the effective potential for a theory with a real scalar field. The effective potential is the energy density of the vacuum in which the expectation value of the scalar field is given by $\langle\phi\rangle=v[13]$. It can be determined from the effective action $\Gamma[\tilde{\phi}]$ according to

$$
\Gamma[\tilde{\phi}=v]=-(2 \pi)^{n} \delta^{(n)}(0) V_{\mathrm{eff}}(v)
$$

where $\tilde{\phi}$ is the vacuum expectation value of the scalar field in the presence of the external source. Using the fact that $\Gamma[\tilde{\phi}]$ is the generating functional of the proper vertex,

$$
\Gamma[\tilde{\phi}]=\sum_{j=1}^{\infty} \frac{1}{j !} \int d^{n} x_{1} d^{n} x_{2} \cdots d^{n} x_{j} \Gamma^{(j)}\left(x_{1}, \cdots, x_{j}\right),
$$

one has

$$
V_{\mathrm{eff}}(v)=-\sum_{j=2}^{\infty} \frac{1}{j !} \Gamma^{(j)}(0,0, \cdots, 0) v^{j},
$$

which means that one can get the effective potential by calculating the 1PI vacuum diagrams. Symmetry breaking occurs when the minimum of the potential occurs at a non-zero value of $v$. In our case we have a complex scalar field $\phi=\chi+i \eta$ and we calculate the effective potential by shifting the real part of the scalar field, $\chi \rightarrow \chi+v$ [13].

A regularization scheme must be chosen to handle the ultraviolet divergences of the theory. In this paper we shall use dimensional regularization. The use of dimensional regularization in a theory that explicitly depends on epsilon tensors involves adopting a complicated form for the gauge field propagator, as will be discussed below. In spite of this complication, dimensional regularization is simpler than Pauli-Villars regularization because of the fact that it allows us to preserve explicit gauge symmetry.

There are several problems involved with analytic continuation to $n$ dimensions. The first of these is standard. In $n$ dimensional space-time the mass dimensions of the fields and parameters are different from their $(2+1)$ dimensional values. In order to arrange for the parameters to keep their original mass dimensions, one introduces a mass scale $\mu$ in such a way that, for each parameter, the dimensional changes are absorbed into a factor $\mu^{(3-n) a}$ where $a$ is a number that depends on the original mass dimensions of the parameter. The second problem is more complicated. Dimensional regularization in a theory with a threedimensional antisymmetric tensor $\epsilon_{\mu \nu \rho}$ must be handled carefully. It has been explicitly shown that naive dimensional regularization schemes cannot make the theory well defined 
when they are applied to a Chern-Simons type model [14. Therefore, in carrying out dimensional regularization we must adopt the three-dimensional analogue of the consistent definition for $\gamma_{5}$, which was originally proposed by 't Hooft and Veltman [15], and later given a strict mathematical justification by Breitenlohner and Maison [16]. The explicit definition of this dimensional continuation for a Chern-Simons-type theory was explained in Ref. [17] where it is shown that this regularization method is indeed compatible with the Slavnov-Taylor identities.

We express the result for the effective potential in terms of the following masses. The scalar masses are,

$$
\begin{aligned}
& m_{\chi}^{2}(v)=m^{2}+\frac{\lambda}{2} v^{2}+\frac{\nu}{24} v^{4} \\
& m_{\eta}^{2}(v)=m^{2}+\frac{\lambda}{6} v^{2}+\frac{v}{120} v^{4}
\end{aligned}
$$

and the gauge boson masses are

$$
\begin{aligned}
& m_{1,2}(v)=\frac{1}{2}\left\{\sqrt{\kappa^{2}+4(e v)^{2}} \pm|\kappa|\right\} \\
& m_{3}^{2}(v):=m_{1}(v) m_{2}(v)
\end{aligned}
$$

The effective potential can be written in three pieces [12]:

$$
V_{\text {eff }}(v)=V_{\text {tree }}+V_{1-l o o p}+V_{2-l o o p}
$$

with

$$
\begin{gathered}
V_{\text {tree }}=\frac{\nu}{6} v^{6} \\
V_{1-\text { loop }}=-\frac{\hbar}{12 \pi}\left\{m_{\chi}^{3}(v)+m_{\eta}^{3}(v)+m_{1}^{3}(v)+m_{2}^{3}(v)\right\} \\
V_{2-\text { loop }}=V_{q 1}+V_{q 2}+V_{c 1}+V_{c 2}+V_{c 3}+V_{c 4} \\
V_{q 1}=\frac{\hbar^{2}}{(4 \pi)^{2}}\left\{3\left(\frac{\lambda}{4}+\frac{15 \nu v^{2}}{6}\right) m_{\chi}^{2}+3\left(\frac{\lambda}{4 !}+\frac{3 \nu v^{2}}{6}\right) m_{\eta}^{2}+2\left(\frac{\lambda}{4}+\frac{9 \nu v^{2}}{6}\right) m_{\chi} m_{\eta}\right\} \\
V_{c 1}=-\hbar^{2}\left[3\left(\frac{\lambda}{3} v+\frac{\nu}{36} v^{3}\right)^{3}+\left(\frac{\lambda}{3} v+\frac{\nu}{60} v^{3}\right)^{2}\right] I_{d i v} \hbar^{2} \mu^{2(n-3)} \frac{\left(m_{\chi}+m_{\eta}\right)\left(m_{1}^{2}+m_{2}^{2}\right)}{m_{1}+m_{2}} \\
+\frac{\hbar^{2}}{16 \pi^{2}}\left\{3\left(\frac{\lambda}{3} v+\frac{\nu}{36} v^{3}\right)^{2} \ln \frac{3 m_{\chi}}{\mu}+\left(\frac{\lambda}{3} v+\frac{\nu}{60} v^{3}\right)^{2} \ln \frac{m_{\chi}+2 m_{\eta}}{\mu}\right\}
\end{gathered}
$$




$$
\begin{aligned}
V_{c 2}= & \frac{e^{2} \hbar^{2}}{2 a}\left[2\left(m_{\chi}^{2}+m_{\eta}^{2}\right)-\left(m_{1}+m_{2}\right)^{2}+3 m_{3}^{2}\right] I_{d i v} \\
+ & \frac{e^{2} \hbar^{2}}{32 \pi^{2} a}\left[\left[m_{\chi} m_{\eta}-\frac{\left(m_{\chi}+m_{\eta}\right)\left(2\left[m_{\chi}-m_{\eta}\right)^{2}+m_{1}^{2}+m_{2}^{2}\right]}{m_{1}+m_{2}}\right]-\frac{\left(m_{\chi}^{2}-m_{\eta}^{2}\right)^{2}}{m_{3}^{2}} \ln \frac{m_{\chi}+m_{\eta}}{\mu}\right. \\
- & \left.\sum_{x=1,2} \frac{2 m_{x}^{2}\left(m_{\chi}^{2}+m_{\eta}^{2}\right)-m_{x}^{4}-\left(m_{\chi}^{2}-m_{\eta}^{2}\right)^{2}}{m_{x}\left(m_{1}+m_{2}\right)} \ln \frac{m_{x}+m_{\chi}+m_{\eta}}{\mu}-\frac{5}{12} \frac{\kappa^{2}}{a^{2}}\right] \\
V_{c 3}= & -\frac{3 \hbar^{2} e^{4} v^{2}}{2 a^{2}} I_{d i v}-\frac{\hbar^{2} e^{4} v^{2}}{32 \pi^{2} a^{2}}\left[3-\frac{2 m_{\chi}}{m_{1}+m_{2}}-\frac{2\left(m_{\chi}^{2}+6 m_{3}^{2}\right)}{\left(m_{1}+m_{2}\right)^{2}}\right] \\
& +\frac{\hbar^{2} e^{4} v^{2}}{64 \pi^{2} a^{2}}\left[\frac{2\left[\left(m_{1}-m_{2}\right)^{2}-m_{\chi}^{2}\right]^{2}}{m_{3}^{2}\left(m_{1}+m_{2}\right)^{2}} \ln \frac{m_{1}+m_{2}+m_{\chi}}{\mu}+\frac{m_{\chi}^{4}}{m_{3}^{4}} \ln \frac{m_{1}}{\mu}\right. \\
& \left.+\sum_{x=1,2}\left[\frac{\left(4 m_{x}^{2}-m_{\chi}^{2}\right)^{2}}{m_{x}^{2}\left(m_{1}+m_{2}\right)^{2}} \ln \frac{2 m_{x}+m_{\chi}}{\mu}-\frac{2\left(m_{x}^{2}-m_{\chi}^{2}\right)^{2}}{m_{3}^{2} m_{x}\left(m_{1}+m_{2}\right)} \ln \frac{m_{x}+m_{\chi}}{\mu}\right]\right]
\end{aligned}
$$

where

$$
I_{\text {div }}=\frac{1}{32 \pi^{2}}\left\{\frac{1}{3-n}-\gamma+1+\ln 4 \pi\right\}
$$

\section{SCALAR QED WITH NON-MINIMAL CHERN-SIMONS COUPLING}

The Lagrangian for scalar QED in $2+1$ dimensions with a non-minimal Chern-Simons coupling is [6]

$$
\mathcal{L}=\frac{1}{2}\left(D_{\mu} \phi\right)^{*} D^{\mu} \phi-\frac{1}{4} F_{\mu \nu} F^{\mu \nu}-\frac{i}{8} \gamma_{0} \epsilon^{\mu \nu \rho} F_{\nu \rho}\left[\phi^{*} D_{\mu} \phi-\left(D_{\mu} \phi\right)^{*} \phi\right]-\frac{\lambda}{6 !}\left(\phi^{*} \phi\right)^{3}
$$

The non-minimal coupling is similar to the minimal Chern-Simons term in several ways: it is odd under parity reversal, topological (in the sense that it does not depend on the space-time metric) and gauge invariant. In spite of the fact that the theory is super non-renormalizable as a consequence of the presence of a coupling constant $\left(\gamma_{0}\right)$ which has negative mass dimension, it can be shown that the Lagrangian is renormalizable to one loop order [6] and that the effective potential is renormalizable to two loop order when certain constraints on the couplings are satisfied [11]. Note that a $\left(\phi^{*} \phi\right)^{2}$ term has not appeared in the Lagrangian

since a term of this form would make a super renormalizable contribution. This term can appear in the renormalized theory however, since the Lagrangian doesn't possess a symmetry that guarantees its vanishing.

We define the following masses:

$$
\begin{aligned}
& m_{\chi}^{2}=\frac{\lambda}{4 !} v^{4} \\
& m_{\eta}^{2}=\frac{\lambda}{5 !} v^{4} \\
& m_{1}=\frac{1}{2} e|v|\left(\sqrt{\left(\gamma_{0} v\right)^{2}+4}+\gamma_{0} v\right),
\end{aligned}
$$




$$
\begin{aligned}
& m_{2}=\frac{1}{2} e|v|\left(\sqrt{\left(\gamma_{0} v\right)^{2}+4}-\gamma_{0} v\right) \\
& m_{3}^{2}=m_{1} m_{2}=e^{2} v^{2}
\end{aligned}
$$

and use the identities

$$
\begin{aligned}
e^{2} & =\frac{1}{v^{2}} m_{3}^{2}, \\
e \gamma_{0} & =\frac{1}{v^{2}}\left(m_{1}-m_{2}\right), \\
\gamma_{0}^{2} & =\frac{1}{v^{2}} \frac{\left(m_{1}-m_{2}\right)^{2}}{m_{1} m_{2}} .
\end{aligned}
$$

The result for the effective potential is

$$
\begin{aligned}
& V(v)=V_{\text {tree }}+V_{1-\text { loop }}+V_{2-l o o p} \\
& V_{\text {tree }}=\frac{\lambda}{6 !} v^{6} \\
& V_{1-\text { loop }}=-\frac{\hbar}{12 \pi}\left[m_{\chi}^{3}+m_{\eta}^{3}+m_{1}^{2}+m_{2}^{2}\right] \\
& V_{2-l o o p}=V_{1}+V_{2}+V_{3}+V_{4}+V_{5} \\
& V_{1}=\frac{\hbar^{2} \lambda v^{2}}{16 \pi^{2}}\left(\frac{3}{2} m_{\chi}^{2}+\frac{3}{10} m_{\eta}^{2}+\frac{1}{10} m_{\chi} m_{\eta}\right) \\
& V_{2}=\frac{\hbar^{2}}{4 \pi^{2}} \frac{1}{v^{2}} \frac{m_{\chi}+m_{\eta}}{m_{1}+m_{2}}\left[\left(m_{1}^{2}-m_{2}^{2}\right)^{2}+2 m_{1}^{2} m_{2}^{2}\right] \\
& V_{3}=\frac{\hbar^{2}}{2}\left(\lambda v^{3}\right)^{2}\left(\frac{1}{3 !^{3}}+\frac{1}{(5 \times 3 !)^{2} \times 2 !}\right) \frac{1}{32 \pi^{2}}\left(\frac{1}{3-n}-\gamma+1+\ln 4 \pi\right) \\
& -\frac{\hbar^{2}}{2}\left(\lambda v^{3}\right)^{2} \frac{1}{32 \pi^{2}}\left[\frac{1}{3 !^{3}} \ln \frac{9 m_{\chi}^{2}}{\mu^{2}}+\frac{1}{(5 \times 3 !)^{2} \times 2 !} \ln \frac{\left(2 m_{\eta}+m_{\chi}\right)^{2}}{\mu^{2}}\right] \\
& V_{4}=V_{4}^{\text {div }}+V_{4}^{\text {finite }} \\
& V_{4}^{d i v}=\frac{\hbar^{2}}{64 \pi^{2}}\left(\frac{1}{3-n}-\gamma+1+\ln (4 \pi)\right)\left(( \frac { 4 } { v ^ { 2 } } + \gamma _ { 0 } ^ { 2 } ) \left(\frac{1}{2}\left(\left(m_{1}-m_{2}\right)^{2}+m_{1} m_{2}\right)\left(m_{\chi}^{2}+m_{\eta}^{2}\right)\right.\right. \\
& \left.\left.-\frac{1}{4}\left(m_{1}^{2}-m_{2}^{2}\right)^{2}+\frac{1}{4} m_{1} m_{2}\left(m_{1}-m_{2}\right)^{2}-\frac{1}{4} m_{1}^{2} m_{2}^{2}\right)-\frac{1}{4} \gamma_{0}^{2}\left(m_{\chi}^{2}-m_{\eta}^{2}\right)^{2}\right) \\
& V_{4}^{\text {finite }}=-\frac{\hbar^{2}}{64 \pi^{2} v^{2}}\left(m_{\chi}-m_{\eta}\right)^{2} \ln \left(\frac{\left(m_{\chi}^{2}+m_{\eta}^{2}\right)^{2}}{\mu^{2}}\right)
\end{aligned}
$$




$$
\begin{aligned}
& +\frac{\hbar^{2}\left(\gamma_{0}^{2} v^{2}+4\right) m_{2}}{256 \pi^{2} v^{2}\left(m_{1}+m_{2}\right)}\left(\left(m_{\chi}+m_{\eta}\right)^{2}-m_{2}^{2}\right)\left(\left(m_{\chi}-m_{\eta}\right)^{2}-m_{2}^{2}\right) \ln \left(\frac{\left(m_{\chi}+m_{2}+m_{\eta}\right)^{2}}{\mu^{2}}\right) \\
& +\frac{\hbar^{2}\left(\gamma_{0}^{2} v^{2}+4\right) m_{1}}{256 \pi^{2} v^{2}\left(m_{1}+m_{2}\right)}\left(\left(m_{\chi}+m_{\eta}\right)^{2}-m_{1}^{2}\right)\left(\left(m_{\chi}-m_{\eta}\right)^{2}-m_{1}^{2}\right) \ln \left(\frac{\left(m_{\chi}+m_{1}+m_{\eta}\right)^{2}}{\mu^{2}}\right) \\
& -\frac{\hbar^{2}\left(\gamma_{0}^{2} v^{2}+4\right)}{1536 \pi^{2} v^{2}\left(m_{1}+m_{2}\right)}\left(12\left(m_{1}^{2}+m_{2}^{2}\right)\left(m_{\chi}^{3}-m_{\chi}^{2} m_{\eta}-m_{\eta}^{2} m_{\chi}+m_{\eta}^{3}\right)+5 m_{1}^{5}+5 m_{2}^{5}\right. \\
& -\left(m_{1}^{3}+m_{2}^{3}\right)\left(10 m_{\chi}^{2}+12 m_{\chi} m_{\eta}+10 m_{\eta}^{2}\right)+m_{3}^{2}\left(m_{1}+m_{2}\right)\left(10 m_{\chi}^{2}+10 m_{\eta}^{2}-5 m_{3}^{2}\right) \\
& \left.+12\left(m_{1}^{4}+m_{2}^{4}\right)\left(m_{\chi}+m_{\eta}\right)\right) \\
& -\frac{\hbar^{2} \gamma_{0}^{2}}{7680 \pi^{2}}\left(6 m_{1}^{4}+6 m_{2}^{4}-\left(12 m_{\eta}^{2}+12 m_{\chi}^{2}+6 m_{3}^{2}\right)\left(m_{1}^{2}+m_{2}^{2}\right)+25 m_{3}^{4}\right. \\
& \left.-26\left(m_{\chi}^{2}+m_{\eta}^{2}\right) m_{3}^{2}+60 m_{\eta} m_{\chi}\left(m_{\chi}^{2}+m_{\eta}^{2}\right)+25\left(m_{\chi}^{2}-m_{\eta}^{2}\right)^{2}\right) .
\end{aligned}
$$

$$
\begin{aligned}
& V_{5}=V_{5}^{e^{2}}+V_{5}^{e \gamma_{0}}+V_{5}^{\gamma_{0}^{2}} \\
& V_{5}^{e^{2}}=-\frac{3 \hbar^{2}}{16 \pi^{2} v^{2}}\left(\frac{1}{3-n}-\gamma+1+\ln (4 \pi)\right) \mu^{(2 n-6)} m_{3}^{4}+\frac{\hbar^{2} m_{\chi}^{4}}{32 \pi^{2} v_{2}} \ln \left(\frac{m \chi^{2}}{\mu^{2}}\right) \\
& -\frac{\hbar^{2} m_{1}\left(m_{2}^{2}-m_{\chi}^{2}\right)^{2}}{16 \pi^{2}\left(m_{1}+m_{2}\right) v^{2}} \ln \left(\frac{\left(m_{2}+m_{\chi}\right)^{2}}{\mu^{2}}\right)-\frac{\hbar^{2} m_{2}\left(m_{1}^{2}-m_{\chi}^{2}\right)^{2}}{16 \pi^{2}\left(m_{1}+m_{2}\right) v^{2}} \ln \left(\frac{\left(m_{1}+m_{\chi}\right)^{2}}{\mu^{2}}\right) \\
& +\frac{\hbar^{2} m_{1}^{2}\left(4 m_{2}^{2}-m_{\chi}^{2}\right)^{2}}{32\left(m_{1}+m_{2}\right)^{2} \pi^{2} v^{2}} \ln \left(\frac{\left(2 m_{2}+m_{\chi}\right)^{2}}{\mu^{2}}\right)+\frac{\hbar^{2} m_{2}^{2}\left(4 m_{1}^{2}-m_{\chi}^{2}\right)^{2}}{32\left(m_{1}+m_{2}\right)^{2} \pi^{2} v^{2}} \ln \left(\frac{\left(2 m_{1}+m_{\chi}\right)^{2}}{\mu^{2}}\right) \\
& +\frac{\hbar^{2} m_{3}^{2}\left(\left(m_{1}-m_{2}\right)^{2}-m_{\chi}^{2}\right)^{2}}{16\left(m_{1}+m_{2}\right)^{2} \pi^{2} v^{2}} \ln \left(\frac{\left(m_{1}+m_{2}+m_{\chi}\right)^{2}}{\mu^{2}}\right) \\
& +\frac{\hbar^{2} m_{3}^{4}\left(-3\left(m_{1}-m_{2}\right)^{2}+2 m_{\chi}\left(m_{1}+m_{2}+m_{\chi}\right)\right)}{8\left(m_{1}+m_{2}\right)^{2} \pi^{2} v^{2}} \\
& V_{5}^{e \gamma_{0}}=-\frac{5}{8 \pi^{2} v^{2}} \hbar^{2}\left(\frac{1}{3-n}-\gamma+1+\ln (4 \pi)\right) \mu^{(2 n-6)}\left(m_{1}-m_{2}\right)^{2} m_{3}^{2} \\
& -\frac{\hbar^{2} m_{3}^{2}\left(m_{1}-m_{2}\right)^{2}\left(49 m_{1}^{2}+49 m_{2}^{2}-42\left(m_{1}+m_{2}\right) m_{\chi}+26 m_{3}^{2}-12 m_{\chi}^{2}\right)}{48\left(m_{1}+m_{2}\right)^{2} \pi^{2} v^{2}} \\
& -\frac{\hbar^{2}\left(4 m_{1}^{2}-m_{\chi}^{2}\right)^{2}\left(m_{2}-m_{1}\right) m_{2}}{16\left(m_{1}+m_{2}\right)^{2} \pi^{2} v^{2}} \ln \left(\frac{\left(2 m_{1}+m_{\chi}\right)^{2}}{\mu^{2}}\right) \\
& +\frac{\hbar^{2}\left(4 m_{2}^{2}-m_{\chi}^{2}\right)^{2}\left(m_{2}-m_{1}\right) m_{1}}{16\left(m_{1}+m_{2}\right)^{2} \pi^{2} v^{2}} \ln \left(\frac{\left(2 m_{2}+m_{\chi}\right)^{2}}{\mu^{2}}\right) \\
& +\frac{\hbar^{2}\left(m_{1}-m_{2}\right)^{2}\left(\left(m_{1}-m_{2}\right)^{2}-m_{\chi}^{2}\right)^{2}}{16\left(m_{1}+m_{2}\right)^{2} \pi^{2} v^{2}} \ln \left(\frac{\left(m_{1}+m_{2}+m_{\chi}\right)^{2}}{\mu^{2}}\right) \\
& +\frac{\hbar^{2}\left(m_{1}-m_{2}\right)\left(m_{2}^{2}-m_{\chi}^{2}\right)^{2}}{16 \pi^{2}\left(m_{1}+m_{2}\right) v^{2}} \ln \left(\frac{\left(m_{2}+m_{\chi}\right)^{2}}{\mu^{2}}\right) \\
& -\frac{\hbar^{2}\left(m_{1}-m_{2}\right)\left(m_{1}^{2}-m_{\chi}^{2}\right)^{2}}{16 \pi^{2}\left(m_{1}+m_{2}\right) v^{2}} \ln \left(\frac{\left(m_{1}+m_{\chi}\right)^{2}}{\mu^{2}}\right)
\end{aligned}
$$




$$
\begin{aligned}
V_{5}^{\gamma_{0}^{2}}= & -\frac{\hbar^{2}\left(m_{1}-m_{2}\right)^{2}}{64 \pi^{2} v^{2}}\left[\mu^{(2 n-6)}\left(\frac{1}{3-n}-\gamma+1+\ln (4 \pi)\right)\left(25\left(m_{1}^{2}+m_{2}^{2}\right)-35 m_{3}^{2}-6 m_{\chi}^{2}\right)\right. \\
& -\frac{2\left(4 m_{1}^{2}-m_{\chi}^{2}\right)^{2}}{\left(m_{1}+m_{2}\right)^{2}} \ln \left(\frac{\left(2 m_{1}+m_{\chi}\right)^{2}}{\mu^{2}}\right)-\frac{2\left(4 m_{2}^{2}-m_{\chi}^{2}\right)^{2}}{\left(m_{1}+m_{2}\right)^{2}} \ln \left(\frac{\left(2 m_{2}+m_{\chi}\right)^{2}}{\mu^{2}}\right) \\
& +\frac{\left(m_{1}^{2}-m_{\chi}^{2}\right)^{2} m_{1}}{\left(m_{1}+m_{2}\right) m_{3}^{2}} \ln \left(\frac{\left(m_{1}+m_{\chi}\right)^{2}}{\mu^{2}}\right)+\frac{\left(m_{2}^{2}-m_{\chi}^{2}\right)^{2} m_{2}}{\left(m_{1}+m_{2}\right) m_{3}^{2}} \ln \left(\frac{\left(m_{2}+m_{\chi}\right)^{2}}{\mu^{2}}\right) \\
& -\frac{\left(m_{1}-m_{2}\right)^{2}\left(\left(m_{1}-m_{2}\right)^{2}-m_{\chi}^{2}\right)^{2}}{\left(m_{1}+m_{2}\right)^{2} m_{1} m_{2}} \ln \left(\frac{\left(m_{1}+m_{2}+m_{\chi}\right)^{2}}{\mu^{2}}\right) \\
& +\frac{1}{2310\left(m_{1}+m_{2}\right)^{2}}\left(89981\left(m_{1}^{4}+m_{2}^{4}\right)-110880 m_{\chi}\left(m_{1}^{3}+m_{2}^{3}\right)\right. \\
& -\left(3837 m_{3}^{2}+28158 m_{\chi}^{2}\right)\left(m_{1}^{2}+m_{2}^{2}\right)+\left(-46200 m_{\chi} m_{3}^{2}+9240 m_{\chi}^{3}\right)\left(m_{1}+m_{2}\right) \\
& \left.\left.-19356 m_{\chi}^{2} m_{3}^{2}+34124 m_{3}^{4}\right)\right]
\end{aligned}
$$

It is straightforward to show that $V_{4}^{\text {div }}$ contains terms that are proportional to $v^{8}$ [1]. As will be discussed in the next section, these terms must be removed for the potential to be renormalizable. We achieve this by imposing the condition

$$
\gamma_{0}^{2}=\frac{(3 \pm \sqrt{5}) \lambda}{60 e^{2}}
$$

In our analysis, we choose $\gamma_{0}=\sqrt{(3+\sqrt{5}) \lambda / 60 e^{2}}$.

\section{ANALYSIS AND RESULTS FOR THE MINIMAL MODEL}

The divergent terms in the effective potential are handled through the renormalization process. They are effectively 'absorbed' by redefining the physical parameters. We throw away the divergent terms, and replace them by a set of counterterms of the form,

$$
V_{c t}=A \frac{v^{2}}{2}+B \frac{v^{4}}{4}+C \frac{v^{6}}{6}
$$

The constants $A, B$, and $C$ are determined, as functions of the parameters of the theory, by imposing the renormalization conditions. A typical choice for these conditions is:

$$
\begin{aligned}
& \left.V\right|_{v=0}=0 \\
& \left.\frac{\partial^{2} V}{\partial v^{2}}\right|_{v=0}=m^{2} \\
& \left.\frac{\partial^{4} V}{\partial v^{4}}\right|_{v=0}=\lambda \\
& \left.\frac{\partial^{6} V}{\partial v^{6}}\right|_{v=\sqrt{M}}=\nu
\end{aligned}
$$

This choice of renormalization conditions is made in [12 and is conventional because the algebra is simpler when $v$ is set to zero in the expressions for the derivatives (the sixth derivative cannot be evaluated at $v=0$ because it is singular there). 
However, in order to ensure that perturbation theory will be reliable, it is advantageous to choose renormalization conditions that give rise to the 'physical' parameters. Towards this end, we choose renormalization conditions of the form,

$$
\begin{aligned}
& \left.V\right|_{v=0}=0 \\
& \left.\frac{\partial^{2} V}{\partial v^{2}}\right|_{v=\sqrt{M}}=m^{2} \\
& \left.\frac{\partial^{4} V}{\partial v^{4}}\right|_{v=\sqrt{M}}=\lambda \\
& \left.\frac{\partial^{6} V}{\partial v^{6}}\right|_{v=\sqrt{M}}=\nu
\end{aligned}
$$

The physical parameters are the ones for which the renormalization point (the point at which the derivatives are evaluated) is the position of the global minimum. The constraint

$$
\left.\frac{\partial V}{\partial v}\right|_{v=\sqrt{M}}=0
$$

ensures that the potential has an extremum at $v=\sqrt{M}$ but not necessarily a global minimum (this extrema could be a local minimum, or a maximim, or an inflection point). To have spontaneous symmetry breaking it must be possible to choose a set of parameters $M, m$, $\lambda$ and $\nu$ so that the couplings are small and the resulting effective potential has a global minimum at $v=\sqrt{M}$. The constraint (34) will give us a condition on the couplings of the form $F(M, m, \lambda, \kappa, \nu)=0$ which we can use to implicitly determine one of the parameters: we will determine $\kappa$ as a function of the other variables. The parameters defined in this way are physical in the sense that they correspond to the parameters of the fundamental excitation that lives in the bottom of the potential well and interacts with the rest of the world through perturbation theory.

If the renormalization point is not chosen to be the position of the global minimum than the parameters defined by (33) and (34) will not be the physical ones. If these 'unphysical' parameters are close to the physical ones then perturbation theory will give approximately the same answer with either choice for the parameters. (If we calculate to all orders in PT then the result will be the same, even if the couplings used are far away from the physical parameters. This is equivalent to saying that the physics is independent of the choice of $M$. However, different choices of $M$ are not equally useful, since in pratice we cannot calculate to all orders in perturbation theory).

Notice that the logic of the renormalization process seems to be exactly backwards: the values of the couplings (or the values of the derivatives at the position of the minima) contain physical information, since these couplings define the shape of the potential at the global minimum. But it is just these couplings that we are choosing. The catch is that we restrict ourselves to choices of couplings that correspond to symmetry breaking. More precisely, we proceed as follows: we start with the physical renormalization conditions (33) and choose some values for $M, m, \lambda$ and $\nu$. We determine $\kappa$ from the derivative constraint (34). (Note that it is equivalent to say that we choose the physical parameters $\kappa, m, \lambda$ and $\nu$ and find the corresponding $M$ ). If it is possible to choose a set of parameters $M, m$, $\lambda$ and $\nu$ so that the couplings are small and the resulting effective potential has a global 
minimum at $v=\sqrt{M}$, then the effective potential gives a perturbatively reliable description of spontaneous symmetry breaking.

We use units in which $\hbar=1$. We set $e=1$ or effectively we rescale parameters to obtain dimensionless variables: $\tilde{v}=v / e, \tilde{M}=M / e^{2}, \tilde{m}=m / e^{2}, \tilde{\lambda}=\lambda / e^{2}$ and $\tilde{\kappa}=\kappa / e^{2}$ and suppress the tildes. We choose values for $M, \nu, m$ and $\lambda$. We use (33) and the value of $\kappa$ determined by the derivative constraint (34). Using the following values,

$$
M=1 ; \quad \nu=0.0005 ; \quad m=.2709 ; \quad \lambda=.2200
$$

we obtain two values of $\kappa$ from the derivative constraint: $\kappa=\{1.6428,20.7494\}$. The resulting potential is very insensitive to the value of $\kappa$. We use the larger value and obtain the two loop potential shown in Fig. [1] which has a global minimum at $v=\sqrt{M}$.

Under certain circumstances, it is advantageous to use the renormalization conditions (32) instead of (33) because the choice (32) is simpler algebraically. However, as discussed above, perturbative calculations using the renormalization conditions (32) are in general less reliable because the couplings differ from the physical values used in the renormalization conditions (33) and (34). As an example, we look at the two loop potential obtained using the renormalization conditions (32) with $m=0, \lambda=0$ and $\nu=.0005$. We impose the constraint (34) to obtain the complete set of parameters,

$$
M=1 ; \quad \nu=0.0005 ; \quad m=\lambda=0 ; \quad \kappa=\{.95757, \quad 25.1850\}
$$

Using the larger value of $\kappa$ we obtain a potential with a global minimum at $v=\sqrt{M}$ as shown in Fig. [2]. We now calculate the values of the second and fourth derivatives at $v=\sqrt{M}$ for the potential in Fig. [2] and obtain respectively $1.014 \times 10^{-6}$ and $5.464 \times 10^{-5}$, which of course differ from the values at the origin. To check the consistency of the calculation, we calculate $\kappa$ using these values with the physical renormalization conditions (B3). The result is 25.2992 which demonstrates good agreement with the value obtained from the renormalization conditions (32) and shows that our numerical calculations are reliable to three digits in this case. Again, we stress that only the values of the derivatives at the true minimum are physical. Moreover, the renormalization conditions (32) are imposed at $v=0$ which is in general far from the true minimum and therefore in a region where the perturbative expansion may not be reliable. We will return to this point later.

In [12] it is claimed that the symmetry breaking disappears when $M$ is changed at the one loop level. The authors then claim that this indicates that perturbation theory is breaking down, and that it is necessary to go beyond one loop. We have shown that this breakdown of perturbation theory occurs when non-physical values of the parameters are used. Fig. [3] shows the one loop effective potential renormalized using (32) with $m=\lambda=0, \kappa=20$, $\nu=0.0005$ and $M=\{.5,1,5\}$. The minima for these graphs are not at $v=\sqrt{M}$ and there is no symmetry breaking for $M=.5$. Fig. [4] shows the one loop potential for the same range of $M$ values using the renormalization conditions (33) and (34). The parameters for the three curves are:

$$
\begin{aligned}
& M=.5, m=.2709, \lambda=.2188, \nu=0.0005, \kappa=1.5588 \\
& M=1, m=.2709, \lambda=.2188, \nu=0.0005, \kappa=\{1.9748414 .2495\} \\
& M=5, m=.2709, \lambda=.2188, \nu=0.0005, \kappa=3.4534
\end{aligned}
$$


In the case where the derivative constraint has two solutions, we choose the smaller value of $\kappa$. As shown in Fig. [4], symmetry breaking persists when the physical parameters are used. Thus we have shown that the result obtained by the authors of [12], that the symmetry breaking depends on the choice of $M$, is an artifact of their choice of renormalization conditions.

Fig. [5] shows the one and two loop potentials for $M=1$ obtained using (33) and (34). On the same graph we have also plotted an expanded expression for the two loop potential. This expansion is useful to clarify the physical content of the potential because the two loop expression is so messy. The expansion parameter is $z=v^{2} / \kappa^{2}$. As long as the minimum occurs at a value of $v$ that corresponds to a small value of $z$, the expanded potential will reliably describe the symmetry breaking. We will show that this expansion is useful under certain conditions: If the renormalization conditions (32) are used, with the choices $m=\lambda=0$, the expansion corresponds to an approximate Coleman-Weinberg limit in the sense that the constraint (34) takes the form of a dimensional transmutation relation which allows us to rewrite the dimensionless parameter $\nu$ in terms of the parameter $\kappa$. Using the renormalization conditions (32) with $m=\lambda=0$ the expanded two loop potential has the form,

$$
V(v)=\frac{\nu v^{6}}{6}+D v^{6}\left(\ln \frac{v}{\sqrt{M}}-\frac{49}{20}\right)
$$

with

$$
D=\frac{1}{32 \pi^{2}}\left(16 \frac{1}{\kappa^{4}}-\frac{11}{30} \frac{\nu}{\kappa^{2}}+\frac{7}{675} \nu^{2}\right)
$$

Imposing the derivative constraint gives the condition

$$
\frac{\nu}{5 !}-\frac{137}{10} D=0
$$

When

$$
\nu=\mathcal{O}\left(\frac{1}{\kappa^{4}}\right)
$$

equations (38) and (39) give,

$$
\nu=\frac{822}{\pi^{2}} \frac{1}{\kappa^{4}}
$$

This expression is a dimensional transmutation relation. In Fig. [5] we use the parameters $M=1, m=.2709, \lambda=.2188$ and $\nu=0.0005$. For the one loop potential we obtain $\{\kappa=1.97484,14.2495\}$; for the two loop potential we have $\{\kappa=1.64451,14.9877\}$; and for the expanded potential $\kappa=10.6287$. We use the large value of $\kappa$ in each case, so that the expansion parameter $z=v^{2} / \kappa^{2}$ is small for $v$ in the vicinity of the global minimum. We note that the two loop potential is extremely sensitive to the value of $m$, and the expanded potential is sensitive to $\lambda$. If we move away from the values chosen above, either the value of $\kappa$ changes drastically, or the derivative constraint (34) has no solution. The figure shows that the three curves are almost identical in the vicinity of the minimum. At large $v$ the curves separate. There is a global minimum at $v=\sqrt{M}$ and thus we have established that, for this choice of parameters, symmetry breaking exists and is perturbatively reliable. 


\section{ANALYSIS AND RESULTS FOR THE NON-MINIMAL MODEL}

When working with the non-minimal model we must impose the constraint (30). This condition between the two parameters $\lambda$ and $\gamma_{0}$ removes the $v^{8}$ terms in the effective potential and is therefore necessary to obtain a renormalizable result. For the non-minimal model we must use renormalization conditions of the same form as those in (33) because the derivatives of the non-minimal potential are divergent at $v=0$. We use

$$
\begin{aligned}
& \left.V\right|_{v=0}=0 \\
& \left.\frac{\partial^{2} V}{\partial v^{2}}\right|_{v=\sqrt{M}}=m^{2} \\
& \left.\frac{\partial^{4} V}{\partial v^{4}}\right|_{v=\sqrt{M}}=\tau \\
& \left.\frac{\partial^{6} V}{\partial v^{6}}\right|_{v=\sqrt{M}}=\lambda
\end{aligned}
$$

Note that we have allowed for the presence of a term of the form $\tau v^{4} / 4$ ! in the renormalized effective potential even though the corresponding term does not appear in the bare Lagrangian. As discussed earlier, a term of this form can appear in the renormalized theory, since the Lagrangian doesn't possess a symmetry that guarantees its vanishing.

We obtain physical couplings by imposing the constraint (34) which gives an equation of the form $f(m, \tau, \lambda, M)=0$. We find solutions to this equation by looking at a range of $M$ 's. For each $M$ we choose values for $m$ and $\tau$ which permit physical solutions to the derivative constraint: values of $\lambda$ which are real and less than one. We further restrict ourselves to $\lambda$ 's which give an effective potential that approaches infinity when $v \rightarrow \infty$ and exhibits symmetry breaking with a global minimum at $v=\sqrt{M}$.

The algebraic expressions for the effective potential are much more complicated for the non-minimal model than for the minimal model. We use an expanded expression for the two loop effective potential to make it easier to identify the choices of parameters that lead to symmetry breaking, and to check that reliable results are produced for the full two loop effective potential. The expansion parameter is $\epsilon=v \gamma_{0}$. This parameter is small in the vicinity of the minimum for all of the examples we look at, as can be seen from Table [1]. In contrast to the case of the minimal model, the expansion does not produce a ColemanWeinberg type dimensional transmutation relation. To understand this point, we remind ourselves how this relation was obtained for the minimal model. The first step was to expand the potential in the parameter $z=v^{2} / \kappa^{2}$. When we imposed (34) on the expanded potential we obtained a result in which all of the logarithm terms disappeared. Furthermore, the variable $M$ dropped out. We were left with a constraint of the form $F(m, \lambda, \nu, \kappa)=0$. To further simplify, we choose $m=\lambda=0$ and obtained a constraint between $\nu$ and $\kappa$. This constraint is the dimensional transmutation relation for the minimal model.

Now compare what happens with the non-minimal model. If we expand in the variable $\epsilon=v \gamma_{0}$ we obtain a constraint from (34) in which the logarithms have disappeared. However, the parameter $M$ does not drop out, and there is one less coupling than in the minimal case because of the constraint (30). We are left with an equation of the form, $f(m, \tau, \lambda, M)=0$. If we set $m=\tau=0$ we find that $\lambda$ is determined for each choice of $M$. 
We compare the one loop, the two loop and the expanded version of the two loop potential, for several values of $M$. The results are shown in Table [1] and Figs. [6-9]. For $M=.5$ no results are given for the expanded potential because in this case the constraint (34) has no solutions for $\lambda$ positive and less than one. The figures show that symmetry breaking exists and perturbation theory is reliable.

\section{Table 1}

\begin{tabular}{lcccccc}
\multicolumn{1}{c}{$\mu=50$} & $\hbar=1$ & $e=1$ & $\gamma_{0}=0.295411 \sqrt{\lambda}$ & \\
\hline Type & M & $\tau$ & $m$ & $\lambda$ & $\gamma_{0}$ & Figure \\
& & & & & & \\
\hline & & & & & & \\
One Loop & 0.5 & 0.88 & 0.20 & 0.5436 & 0.2178 & Fig. 6 \\
Two Loop & & & & 0.1454 & 0.1126 & \\
& & & & & & \\
One Loop & 1 & 0.67 & 0.25 & 0.2226 & 0.1394 & Fig. 7 \\
Expanded & & & & 0.5472 & 0.2185 & \\
Two Loop & & & & 0.0794 & 0.0832 & \\
& & & & & & \\
One Loop & 5 & 0.42 & 0.55 & 0.0219 & 0.0437 & Fig. 8 \\
Expanded & & & & 0.0301 & 0.0512 & \\
Two Loop & & & & 0.0176 & 0.0391 & \\
& & & & & & \\
One Loop & 10 & 0.30 & 0.60 & 0.0147 & 0.0358 & Fig. 9 \\
Expanded & & & & 0.0146 & 0.0357 & \\
Two Loop & & & & 0.0114 & 0.0315 &
\end{tabular}

Note that although the shapes of the wells at the true minima are similar for the one loop and two loop potentials, the depths of the wells are quite different. To understand this behaviour we recall that the value of the effective potential at the true minimum depends on the renormalization condition $\left.V\right|_{v=0}=0$. The important point is that this condition is imposed at $v=0$ which is in general far from the position of the true minimum. The only physical quantities that are perturbatively reliable are those which depend on the properties of the effective potential near the true minimum, namely $\tau, m$ and $\lambda$ (or $\gamma_{0}$ ). The first two parameters are fixed by the renormalization conditions both at one loop and two loop. The true test of the reliability of the perturbative expansion comes from comparing the values of $\gamma_{0}$ obtained from (34) for the one and two loop potentials. Table [1] shows that these values compare fairly well, especially for higher values of $M$. 


\section{CONCLUSIONS}

We have studied spontaneous symmetry breaking for $2+1$ dimensional scalar QED with both minimal and non-minimal Chern Simons couplings. The effective potential for both of these models has been calculated previously [11,12]. Starting from these expressions, we have studied the renormalization procedure in an attempt to shed light, perturbatively, on the nature of the symmetry breaking in both models. All calculations have been carried out using Mathematica.

First, we have looked for symmetry breaking at one and two loops in the minimal model using the physical renormalization point (which means defining the couplings in terms of the derivatives of the effective potential at the global minimum). We have obtained a renormalized effective potential that exhibits spontaneous symmetry breaking (for an appropriate choice of couplings) and is perturbatively reliable. Our main results for the minimal model are summarized in Figs. [4] and [5]. Fig. [4] shows that symmetry breaking at one loop is independent of the choice of the renormalization point. Fig. [5] shows that two loop corrections near the minimum are small. These results disagree with the those of [12] in which it was found that the presence of symmetry breaking at one loop depends on the choice of the renormalization point. We have shown that this result is a consequence of using a non-physical renormalization point.

Secondly, we have performed a two loop analysis of the non-minimal model. We use the physical renormalization point and obtain an effective potential that exhibits spontaneous symmetry breaking (for an appropriate choice of couplings) and is perturbatively reliable. The renormalization conditions are considerably more complicated for the nonminimal model, in part because of the more complicated structure of the interaction, and in

part because of the fact that all of the derivatives of the effective potential are singular at the origin. We were not able to obtain a simple dimensional transmutation relation for the non-minimal model.

\section{ACKNOWLEDGMENTS}

This work is supported by the Natural Sciences and Engineering Research Council of Canada. 


\section{REFERENCES}

[1] S. Deser, R. Jackiw and S. Templeton, Ann. Phys. (N.Y.) 140 (1982) 372; R. Jackiw and S. Templeton, Phys. Rev. D23 (1983) 2291.

[2] F. Wilczeck (ed.), Fractional Statistics, Superconductivity of Anyons (World Scientific, Singapore, 1990); S. Forte, Rev. Mod. Phys. 64 (1992) 193.

[3] J. Hong, Y. Kim and P.Y. Park, Phys. Rev. Lett. 64 (1990) 2230; R. Jackiw and E.J. Weinberg, Phys. Rev. Lett. 64 (1990) 2234; R. Jackiw, K. Lee and E. Weinberg, Phys. Rev. D42 (1990) 3488.

[4] D. Gross, R. Pisaski and L. Yaffe, Rev. Mod. Phys. 53 (1981) 43; R. Efraty and V.P. Nair, Phys. Rev. Lett. 68 (1992) 2891.

[5] J. Goryo and K. Ishikawa, Phys. Lett A (1998) 246.

[6] M.E. Carrington and G. Kunstatter, Phys. Rev. D50 (1994) 2830.

[7] M.E. Carrington and G. Kunstatter, Phys. Lett. B321, 3836 (1993); M.E. Carrington and G. Kunstatter, Phys. rev. D51, 1903 (1995).

[8] S. Coleman and E. Weinberg, Phys. Rev. D7 (1973) 1888.

[9] A. Antilloón, J. Escalona and M. Torres, Phys. Rev. D55 (1997) 6327.

[10] M. Torres, Phys. Rev. D46 (1992) R2295.

[11] M.E. Carrington, W.F. Chen, G. Kunstatter and J. Mottershead, Phys. rev. D60, 125018 (1999).

[12] P.N. Tan, B. Tekin and Y. Hosotani, Phys. Lett. B388 (1996) 611; Nucl. Phys. B502 (1997) 483.

[13] S. Coleman, Aspects of Symmetry, Chapter 5 (Cambridge University Press, 1985).

[14] M. Chaichian and W.F. Chen, Phys. Rev. D58 (1998) 125004.

[15] G. 't. Hooft and M. Veltman, Nucl. Phys. B44 (1972) 189.

[16] P. Breitenlohner and D. Maison, Comm. Math. Phys. 52 (1977) 11.

[17] G. Giavarini, C.P. Martin and F. Ruiz Ruiz, Nucl. Phys. B381 (1992) 222. 


\section{FIGURES}

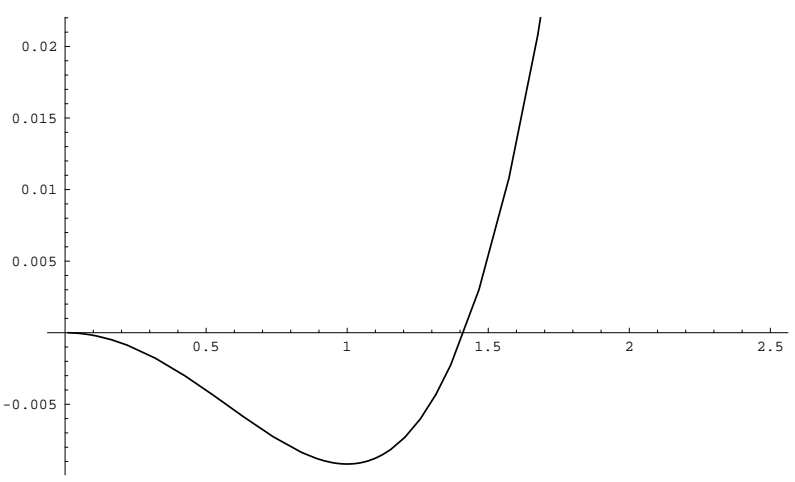

FIG. 1. The two loop minimal effective potential using the renormalization conditions (33) and (34) with $M=1, m=.2709, \lambda=.2200, \nu=0.0005$ and $\kappa=20.7494$.

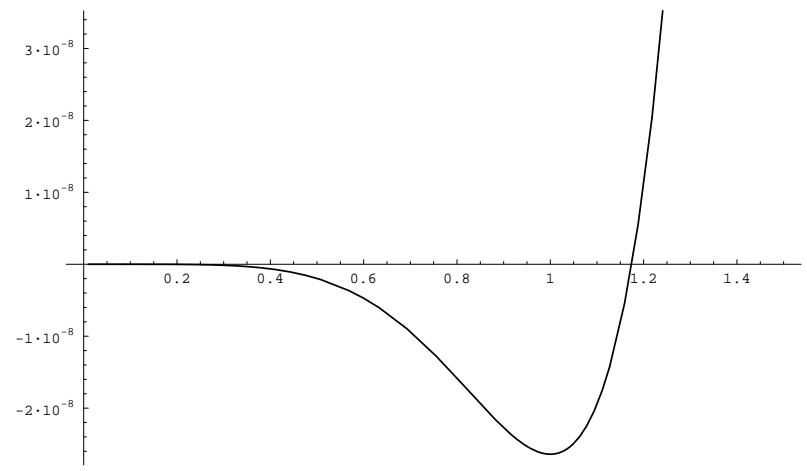

FIG. 2. The two loop minimal effective potential using the renormalization conditions (32) and (34) with $M=1, m=\lambda=0, \nu=0.0005$ and $\kappa=25.1850$. 

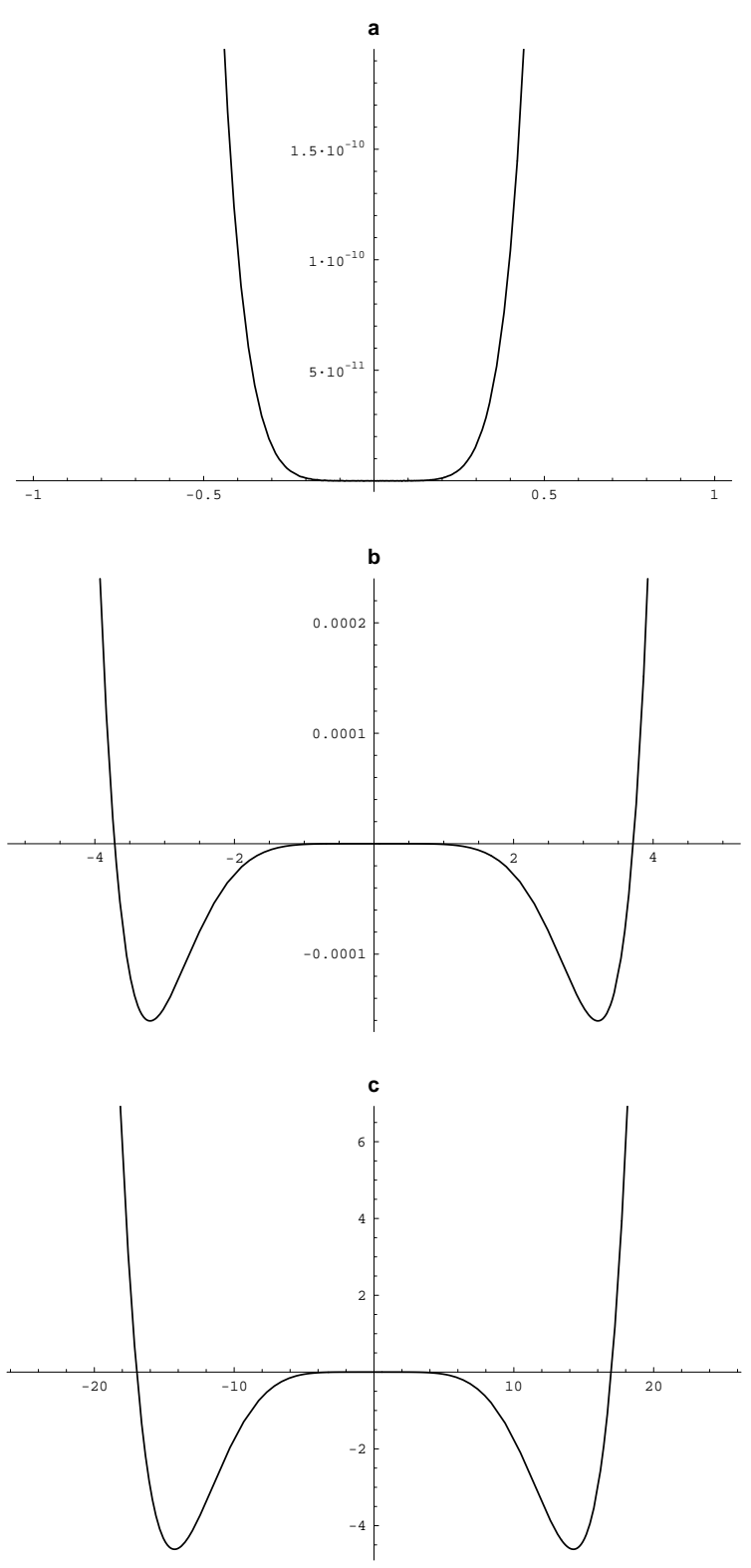

FIG. 3. The one loop minimal effective potential using the renormalization conditions (32) with $m=\lambda=0, \nu=0.0005, \kappa=20$ and (a) $M=.5$; (b) $M=1$; (c) $M=5$. The minima for these graphs are not at $\sqrt{M}$. There is no symmetry breaking for $M=.5$. 


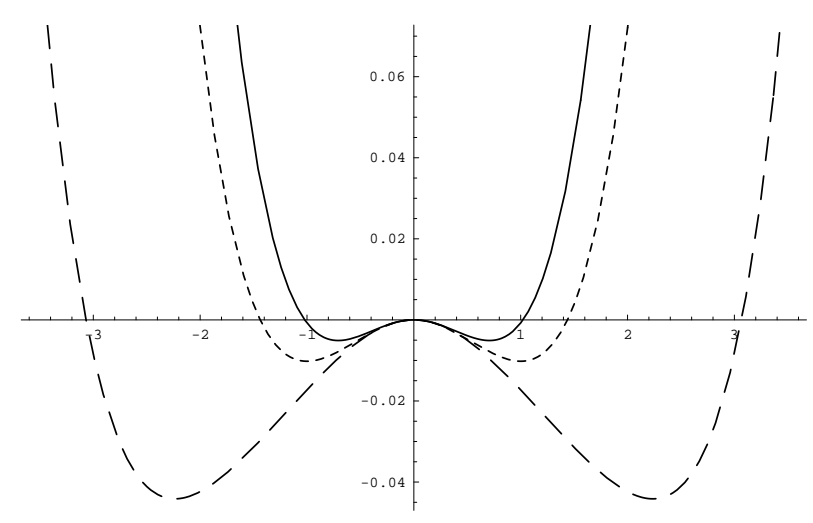

FIG. 4. The one loop minimal effective potential using the renormalization conditions (33) and (34) with $m=.2709, \lambda=.2188, \nu=0.0005$ and $M=.5, \kappa=1.5588$ (solid line); $M=1$, $\kappa=1.9748$ (dotted line); $M=5, \kappa=3.4534$ (dashed line).
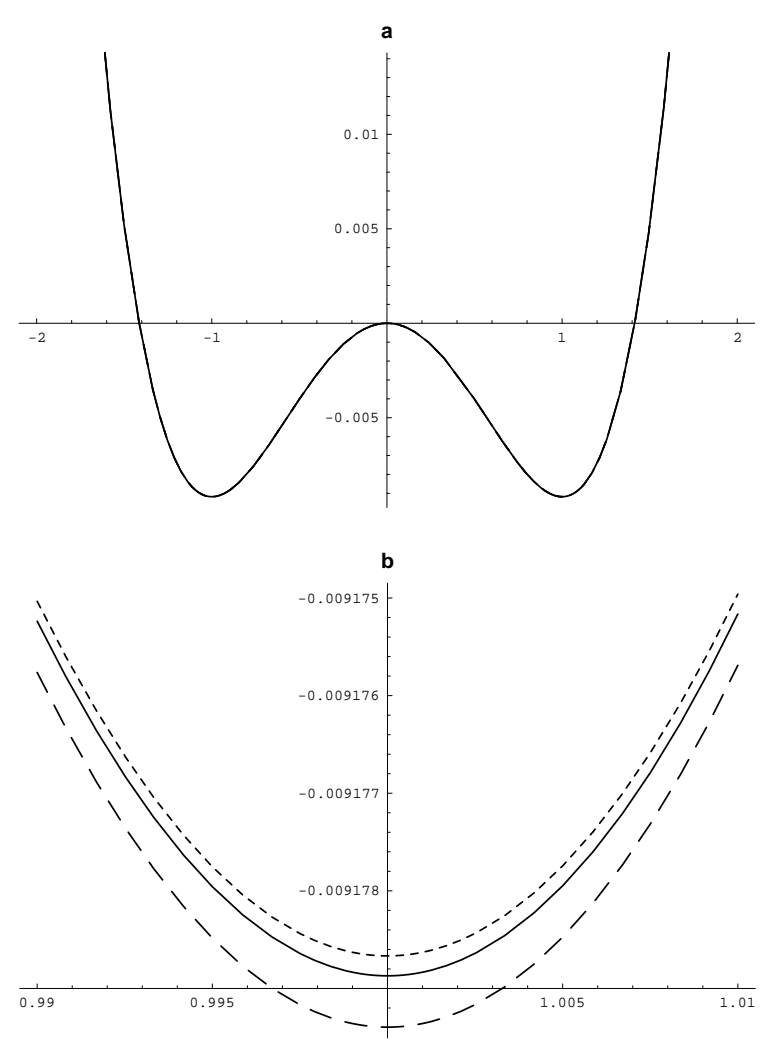

FIG. 5. The minimal effective potential using the renormalization conditions (33) and (34) with $M=1, m=.2709, \lambda=.2188$ and $\nu=0.0005$. The values of $\kappa$ are: one loop $\kappa=14.2495$ (dotted line); two loop $\kappa=14.9877$ (solid line); expanded $\kappa=10.6287$ (dashed line). 


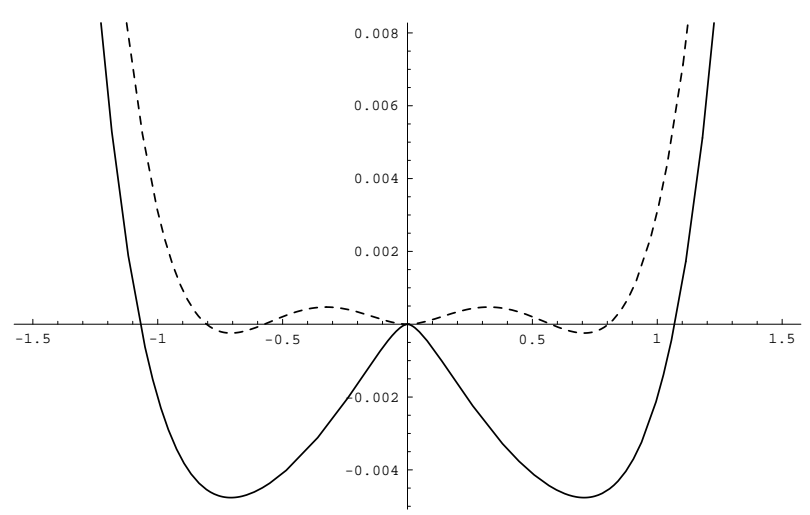

FIG. 6. Non-minimal effective potential for $M=.5$ and other parameters as given in Table [1]. The dotted line is the one loop result and the solid line corresponds to two loops.

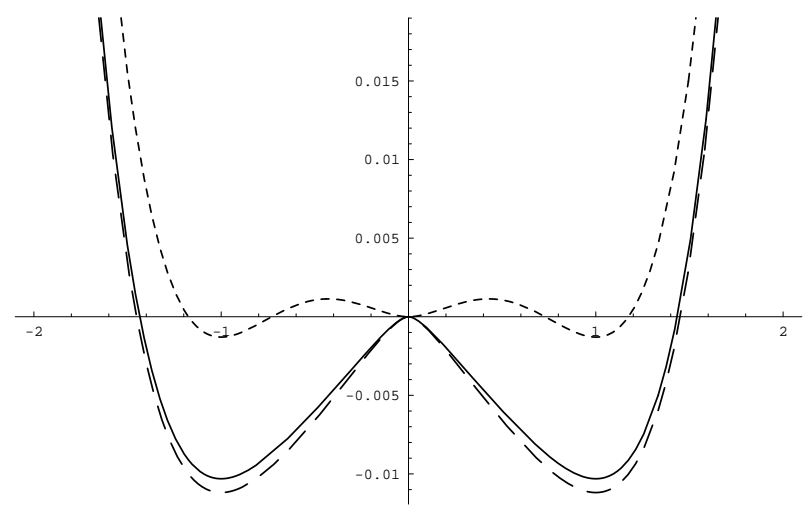

FIG. 7. Non-minimal effective potential for $M=1$ and other parameters as given in Table [1]. The dotted line is the one loop result, the solid line corresponds to two loops, and the dashed line is the expanded result.

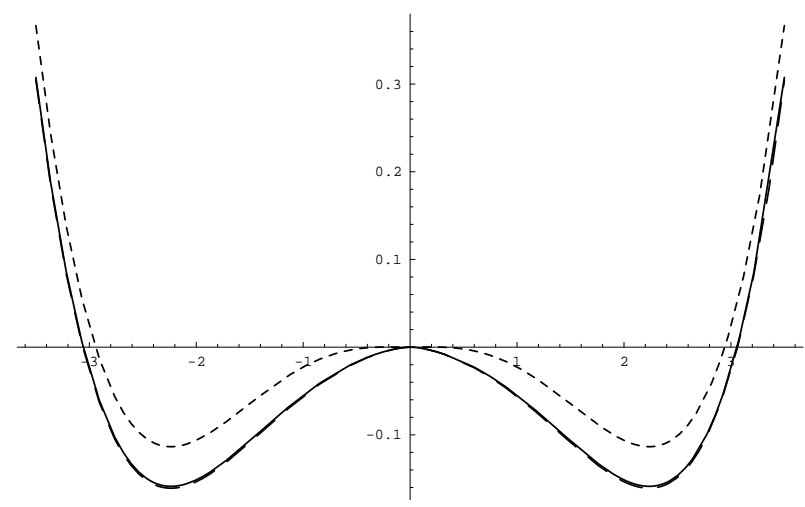

FIG. 8. Non-minimal effective potential for $M=5$ and other parameters as given in Table [1]. The dotted line is the one loop result, the solid line corresponds to two loops, and the dashed line is the expanded result. 


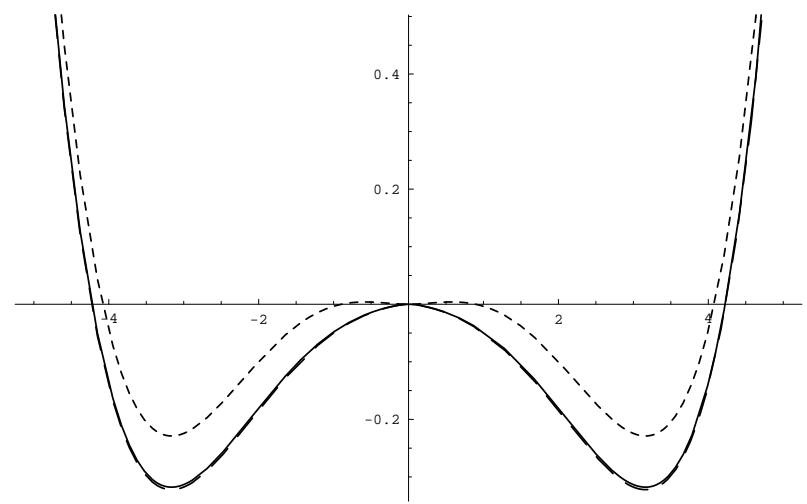

FIG. 9. Non-minimal effective potential for $M=10$ and other parameters as given in Table [1]. The dotted line is the one loop result, the solid line corresponds to two loops, and the dashed line is the expanded result. 